\title{
The Aged Epidermal Permeability Barrier Structural, Functional, and Lipid Biochemical Abnormalities in Humans and a Senescent Murine Model
}

Ruby Ghadially, ${ }^{\star \ddagger}$ Barbara E. Brown, ${ }^{\ddagger}$ Sandy M. Sequeira-Martin, ${ }^{\ddagger}$ Kenneth R. Feingold, ${ }^{\star \star}$ and Peter M. Elias ${ }^{\star *}$

*Department of Dermatology, University of California, San Francisco, San Francisco, California 94143; and ${ }^{\ddagger}$ Dermatology Service, Department of Veterans Affairs Medical Center, San Francisco, California 94121

\begin{abstract}
Aged epidermis displays altered drug permeability, increased susceptibility to irritant contact dermatitis, and often severe xerosis, suggesting compromise of the aged epidermal barrier. To delineate the functional, structural, and lipid biochemical basis of epidermal aging, we compared barrier function in young (20-30 $\mathrm{yr})$ vs aged $(>80 \mathrm{yr})$ human subjects, and in a murine model. Baseline transepidermal water loss in both aged humans and senescent mice was subnormal. However, the aged barrier was perturbed more readily with either acetone or tape stripping $(18 \pm 2$ strippings vs $31 \pm 5$ strippings in aged vs young human subjects, respectively). Moreover, after either acetone treatment or tape stripping, the barrier recovered more slowly in aged than in young human subjects $(50$ and $80 \%$ recovery at 24 and $72 \mathrm{~h}$, respectively, in young subjects vs $15 \%$ recovery at $24 \mathrm{~h}$ in aged subjects), followed by a further delay over the next $6 \mathrm{~d}$. Similar differences in barrier recovery were seen in senescent vs young mice. Although the total lipid content was decreased in the stratum corneum of aged mice $(\sim 30 \%)$, the distribution of ceramides (including ceramide 1), cholesterol, and free fatty acids was unchanged. Moreover, a normal complement of esterified, very longchain fatty acids was present. Finally, stratum corneum lamellar bilayers displayed normal substructure and dimensions, but were focally decreased in number, with decreased secretion of lamellar body contents. Thus, assessment of barrier function in aged epidermis under basal conditions is misleading, since both barrier integrity and barrier repair are markedly abnormal. These functional changes can be attributed to a global deficiency in all key stratum corneum lipids, resulting in decreased lamellar bilayers in the stratum corneum interstices. This constellation of findings may explain the increased susceptibility of intrinsically aged skin to exogenous and environmental insults. (J. Clin. Invest. 1995. 95:2281-2290.) Key words: stratum corneum • transepidermal water loss $\bullet$ electron microscopy $\bullet$ lipids
\end{abstract}

\section{Introduction}

Physiological changes in intrinsically (chronologically) aged skin can produce significant morbidity in the aging population,

\footnotetext{
Address correspondence to Ruby Ghadially, Dermatology Service (190), Veterans Affairs Medical Center, 4150 Clement Street, San Francisco, CA 94121. Phone: 415-750-2091; FAX: 415-751-3927.

Received for publication 24 May 1994 and in revised form 5 January 1995.
}

The Journal of Clinical Investigation, Inc.

Volume 95, May 1995, 2281-2290 including altered drug permeability, increased susceptibility to irritant contact dermatitis, and often severe xerosis. In recent years considerable attention has focused on functional abnormalities of the dermis in intrinsically and photoaged skin. The relative lack of interest in the epidermis of the aged may reflect the reportedly normal thickness of the stratum corneum (1-6), with an increase in corneocyte surface area the only described structural abnormality (7). Likewise, only minor functional abnormalities have been reported in aged epidermis. Stratum corneum water content is reportedly normal $(8-11)$ or slightly decreased (12), and only slight differences in barrier function have been reported both in vitro (1) and in vivo $(9,13,14)$. Yet, drug permeability appears to be significantly altered; for example, the in vitro permeability of water-soluble dyes, such as fluorescein, is increased (15), while the permeability of lipidsoluble drugs, such as testosterone, is decreased (16). These differences in basal transepidermal water loss (TEWL) ${ }^{1}$ vs drug permeability suggest that a barrier abnormality may exist in chronologically aged epidermis, which is masked by the seemingly normal measurements of basal TEWL. Although any differences in permeability should be explicable by changes in stratum corneum lipid composition (17), prior studies have provided conflicting results about the lipid composition of aging human stratum corneum $(18,19)$. These conflicting results reflect data derived from sampling of surface lipids $(18,19)$, which may not accurately reflect stratum corneum lipid content (20), or from assessment of an incomplete spectrum of epidermal-derived lipids (19).

Since functional abnormalities in other senescent organs often only become evident when the tissue is either stressed or required to carry out repair processes $(21-23)$, our strategy has been to assess the barrier in intrinsically aged epidermis, not only under basal conditions, but also when the epidermis is subjected to stress; i.e., barrier repair after experimental abrogation. In young epidermis barrier abrogation is followed by a repair process that includes: $(a)$ immediate secretion of preformed epidermal lamellar bodies; $(b)$ replenishment of the cytosol with newly formed lamellar bodies and further secretion of lamellar body contents; and ( $c$ ) formation of lamellar bilayers in the stratum corneum interstices leading to recovery of the barrier to transepidermal water loss within $24 \mathrm{~h}$. Epidermal lipid synthesis is required to sustain this process (13) and DNA synthesis is also regulated by barrier requirements (24). The increase in lipid synthesis is due to increased activities of key enzymes of cholesterol synthesis, 3-hydroxy-3-methylglutaryl CoA (HMG CoA) reductase (25), ceramide synthesis, serine palmitoyl transferase (26), and fatty acid synthesis, acetyl CoA carboxylase and fatty acid synthetase (27).

1. Abbreviations used in this paper: HMG CoA, 3-hydroxy-3-methylglutaryl CoA; TEWL, transepidermal water loss. 
We therefore compared epidermal barrier function in aged ( $>80 \mathrm{yr}$ ) vs young $(20-30 \mathrm{yr}$ ) human subjects as well as in a new murine model of skin senescence. We describe here that both barrier integrity (ability to withstand perturbation) and barrier recovery after acute perturbations are altered in aging epidermis, despite normal or subnormal basal TEWL rates. These changes in function can be ascribed to abnormalities in lipid content, which in turn, result in insufficient formation of intercellular lamellar bilayers in senescent stratum corneum.

\section{Methods}

\section{Human and animal studies}

Human volunteers. 21 human volunteers, 6 older than $80 \mathrm{yr}$ and 15 younger than $30 \mathrm{yr}$ gave informed consent to participate in these studies. Volunteers were in good general health and free from metabolic diseases, such as diabetes, thyroid disease, or hyperlipidermia, as well as atopic dermatitis. None were taking oral corticosteroids, nonsteroidal antiinflammatory drugs, or oral hypoglycemic agents.

Mice. Hairless mice outbred CrL:SKHI (hr/hr) BR (Charles River Laboratories, Wilmington, MA) were used for the senescent murine model because the other commercially available strains that we tested frequently developed tumors and died before the development of chronological senescence. Young mice were between 6 and $10 \mathrm{wk}$ of age, and aged mice were 18-24 mo old at the time of study (normal life expectancy of hairless mice is $\sim 24 \mathrm{mo}$ ). The aged mice were checked regularly for microbial diseases and tumors. Animals that showed evidence of either systemic illness or tumor development were not studied.

\section{Functional studies}

Basal TEWL (human and murine). Basal TEWL was assessed at three sites on the volar forearm of the human volunteers, using an evaporimeter (Servomed ${ }^{\star}$, Vällingby, Sweden) and recorded in $\mathrm{g} / \mathrm{m}^{2}$ per $\mathrm{h}(28)$. TEWL also was determined over three different sites in seven-young and seven-senescent mice using an electrolytic water analyzer (Meeco, Inc., Warrington, PA), and recorded in $\mathrm{ppm} / 0.5 \mathrm{~cm}^{2}$ per $\mathrm{h}$, and subsequently converted to $\mathrm{g} / \mathrm{m}^{2}$ per $\mathrm{h}$. Temperature ranged between $20^{\circ}$ and $22^{\circ} \mathrm{C}$, relative humidity between 42 and $49 \%$, and atmospheric pressure between $9.4-12.6 \mathrm{mmHg}$ at the time of measurements.

Barrier integrity. Baseline TEWL measurements were made at each of these sites on the volar forearm using the evaporimeter, and each site then was stripped sequentially with cellophane (Tuck; Tesa Tuck Inc., New Rochelle, NY) tape. TEWL was assessed over the same sites after each five tape strippings. Barrier integrity (the ability of the stratum corneum to resist barrier perturbation) is reflected by the number of tape strippings required to attain TEWL levels of $\geq 20 \mathrm{~g} / \mathrm{m}^{2}$ per $\mathrm{h}$.

Barrier recovery experiments (human). A site $\sim 4 \mathrm{~cm}$ below the antecubital fossa on the volar forearm of both aged and young subjects was used for recovery studies. One arm was treated with acetone-soaked cotton balls, while the same sites on the contralateral forearm served as the control. We also used recovery after tape stripping as a second method to control for possible toxic effects of acetone. TEWL was determined $0,3,6,24,48,72$, and sometimes $96 \mathrm{~h}$ after barrier disruption.

Barrier recovery experiments (murine). One flank was treated with acetone-soaked cotton balls or with sequential tape stripping, as described above, and TEWL was measured $0,3,6,24$, and $48 \mathrm{~h}$ after barrier abrogation. Skin biopsies were obtained at each of these time points from parallel groups of similarly treated animals for the histochemical studies described below, as well as for electron microscopy with reduced osmium tetroxide as described below.

Data shown are mean \pm SE. Statistical significances were determined using an unpaired Student's $t$ test.

Electron microscopy. Shave biopsies, obtained under local anesthesia, were taken from the normal appearing, non-sun-exposed, volar forearm or thigh skin of five of the aged $(>80 \mathrm{yr})$ and eight of the young $(<30 \mathrm{yr})$ human volunteers. Prior studies have shown that thigh and forearm display similar barrier repair kinetics (29). Samples were fixed in half-strength Karnovsky's fixative, divided, and processed routinely through either reduced $1.0 \%$ osmium tetroxide or $0.25 \%$ ruthenium tetroxide (30), followed by embeddment in an Epon-epoxy mixture (31). Full thickness biopsies from the flanks of both aged ( $>18$ mo), and young (6-10 wk old) hairless mice also were processed, as described above. Ultrathin sections were viewed in an electron microscope (10A; Carl Zeiss, Inc., Thornwood, NY) after further contrasting in lead citrate and uranyl acetate. The quantities of lamellar bodies in the cytosol were quantitated by standard stereological methods (32), utilizing both randomly obtained sections and blinded data collection.

Histochemical localization of neutral lipids. A saturated solution of sudan red 7B in 70\% ethanol was used to stain for neutral lipids. 4- $\mu \mathrm{m}$ fresh frozen cryostat sections were allowed to dry on the microscope slide and then the slides were transferred to the stain for $10 \mathrm{~min}$ at room temperature, followed by sequential rinsing in $70 \%$ ethanol and distilled water (33). The sections then were counterstained in hematoxylin for $30 \mathrm{~s}$ at room temperature, rinsed in distilled water, and mounted for microscopic examination. For fluorescence localization of lipids a stock solution of nile red $(500 \mu \mathrm{g} / \mathrm{ml})$ in acetone was prepared, stored at $-20^{\circ} \mathrm{C}$, and protected from light. A fresh staining solution of nile red was prepared by the addition of $15-20 \mu \mathrm{l}$ of the stock solution per 1 ml 75\% glycerol followed by brisk vortexing. Fresh frozen cryostat sections were stained, covered with a glass coverslip, and examined after $10 \mathrm{~min}$ at room temperature in the dark, with a fluorescent microscope (Ortholux II; E. Leitz, Inc., Rockleigh, NJ), using 450-nm excitation and 500-nm emission frequencies (34).

Lipid content analysis. Full-thickness murine skin from both age groups was heat split at $60^{\circ} \mathrm{C}$ for $1 \mathrm{~min}$ to remove intact epidermis from dermis. Nucleated epidermal cells then were removed from the stratum corneum by floating the full-thickness skin, basal side downward, in $0.5 \%$ trypsin in PBS for $30 \mathrm{~min}$, followed by vortexing. This method leaves the residual stratum corneum sheet free of nucleated cells (35, 36 ). Both the stratum corneum wet weight, as well as the initial surface area were recorded, and used to calculate yields. The lipids were extracted by the method of Bligh and Dyer (37), dried, weighed, and stored at $-70^{\circ} \mathrm{C}$ until analyzed. Lipids were fractionated and quantified by high performance thin layer chromatography (HPTLC) followed by charring and scanning densitometry, using a modification of the method described by Ponec et al. (38). Lipids were solubilized in chloroformmethanol (2:1 vol) and spotted on HPTLC plates (E. Merck, Darmstadt, Germany) using a CAMAG Linomat IV Autospotter (CAMAG Scientific Inc., Wilmington, NC). The plates were developed in CAMAG horizontal developing chambers at $4^{\circ} \mathrm{C}$. Neutral lipids were resolved using two solvent systems. The plates first were developed three times in petroleum ether:diethyl ether:acetic acid $(80: 20: 1 \mathrm{vol})$ at $4^{\circ} \mathrm{C}$ for 30 $\mathrm{mm}, 50 \mathrm{~mm}$, and $70 \mathrm{~mm}$, respectively, and then developed once in petroleum ether alone or up to $98 \mathrm{~mm}$. Ceramides and glucosylceramides were resolved using first chloroform/methanol/acetone/acetic acid (76:20:4:1 vol) for $20 \mathrm{~mm}$, then chloroform/methanol/acetone (80:10:10 vol) for $50 \mathrm{~mm}$, and finally chloroform/diethylether/ethylacetate/methanol (76:6:20:2 vol) for $98 \mathrm{~mm}$. After development the plates were dipped in a charring solution of glacial acetic acid/concentrated sulfuric acid/ortho-phosphoric acid/distilled water (1:0.2:0.2:1.9 vol) containing $1.5 \% \mathrm{wt} / \mathrm{vol}$ copper sulphate, drained briefly, and then dried for $10 \mathrm{~min}$ on a $50^{\circ} \mathrm{C}$ hot plate. Plates then were transferred to an $180^{\circ} \mathrm{C}$ oven for $15 \mathrm{~min}$. After cooling to room temperature, the plates were scanned immediately and quantitated using a automated TLC Scanner II equipped with a Laboratory Data System and "CATS" software (both from CAMAG Scientific Inc.). Statistical significances were determined using a Student's two-tailed $t$ test.

Gas liquid chromatography of epidermal lipids. Full-thickness skin from senescent hairless male mice was collected, the epidermis was split from the underlying skin by exposure to dry heat $\left(60^{\circ} \mathrm{C}\right.$ for $\left.60 \mathrm{~s}\right)$, and the epidermal lipids extracted with Bligh/Dyer solvents, as above. Epidermal lipids were subfractionated by HPTLC in petroleum ether/ diethyl ether/acetic acid (80:20:1 vol), as described above. The neutral 
lipids were pooled and subsequently methylated in boron trichloride $(12 \% \mathrm{wt} / \mathrm{wt})$ overnight at $80^{\circ} \mathrm{C}$. The pooled methyl ethers were resolved and quantitated on a capillary column $30 \mathrm{~m} \times 0.25 \mathrm{~mm}$ inner diameter, $0.2 \mu \mathrm{M}$ film (SP2380; Supelco Inc., Bellefonte, PA) in a gas chromatograph (5890; Hewlett-Packard Co., Palo Alto, CA), equipped with an autosampler, under the following conditions: column temperature $8 \mathrm{~min}$ at $150^{\circ} \mathrm{C}$ then increased by $3^{\circ} \mathrm{C} / \mathrm{min}$ to $190^{\circ} \mathrm{C}$ and held for $30 \mathrm{~min}$; linear velocity $20 \mathrm{~cm} / \mathrm{s}$; vector type: FID $\left(2 \times 10^{11}\right.$ attenuated full spectrum); split ratio: 100:1; 9 psi head pressure. Peaks were identified against authentic methyl ester standards from Supelco or fatty acids from Sigma Chemical Co. (St. Louis, MO), methylated as described above.

\section{Results}

\section{Structure, function, and lipid composition of aged} epidermis under basal conditions

Basal barrier function. We first determined whether aged epidermis displays abnormalities in barrier function under basal conditions. Under basal conditions, aged human skin appeared paler, with a rougher, drier appearing surface than in the young cohort. In comparison to young mice, aged mice displayed a thickened, yellow appearing integument, with a coarse, dry surface, as well. In both humans and in mice, basal transepidermal water loss rates were slightly decreased in aged vs younger individuals. Mean TEWL rates in aged humans were $4.43 \pm 0.16$ $\mathrm{g} / \mathrm{m}^{2}$ per $\mathrm{h}$ vs a mean of $6.41 \pm 0.93 \mathrm{~g} / \mathrm{m}^{2}$ per $\mathrm{h}$ in the young $(P<0.05)$. Likewise, basal TEWL rates were decreased in aged vs young mice (aged: $0.24 \pm 0.07 \mathrm{~g} / \mathrm{m}^{2}$ per $\mathrm{h}$ vs young: $0.74 \pm 0.13 \mathrm{~g} / \mathrm{m}^{2}$ per $\mathrm{h} ; P<0.01$ ). These results demonstrate that barrier function, assessed by TEWL, is not deficient in intrinsically aged skin under basal conditions.

Stratum corneum membrane structure and the lamellar body secretory system. We next evaluated the quantities and internal structure of the lamellar body and its secreted contents in aged epidermis. Electron microscopy of the epidermis of young humans and mice appeared similar, with comparable numbers of lamellar bodies in the granular cell cytosol, and abundant secreted lamellar body contents at the stratum granulosum-stratum corneum interface (Fig. $1 F$, human). Moreover, as previously described (30), extensive multilamellar arrays, displaying a characteristic basic unit structure, were present at all levels of the stratum corneum interstices (Fig. $1 G$, human).

In aged human and mouse epidermis both the internal structure of lamellar bodies as well as their numbers in the granular cell cytosol were similar to young subjects (Fig. $1 E$, mouse). Stereological measurements showed that lamellar bodies occupied $5.0 \pm 0.4$ vs $5.1 \pm 0.6$ (mean \pm SEM; $n=8$ samples each) percent of the granular cell cytosol in young vs old human epidermis, respectively. However, a paucity of secreted lamellar body contents was present at the stratum granulosum-stratum corneum interface in aged epidermis (Fig. 1, $C$ [mouse] and $D$ [human]). Moreover, the stratum corneum interstices contained focal domains that were either depleted or virtually devoid of lamellar structures (Fig. 1, $A$ and $B$, human). Yet, where the lamellar bilayers were present in either normal or diminished numbers, the lamellar arrays themselves displayed both normal substructure and dimensions (interlamellar dimension [humans]: young $=13.2 \mathrm{~nm}$; aged $=13.3 \mathrm{~nm})$. These results demonstrate that aged epidermis displays a diminution in secreted lamellar body-derived contents, and a failure of these contents to form a continuous series of multilamellar bilayers within the intercellular spaces of the stratum corneum.
Lipid composition of aged murine stratum corneum. To delineate the lipid biochemical basis for the structural abnormalities, we next compared both the lipid content and the lipid distribution of senescent vs young murine stratum corneum. Lipid extracts demonstrated a significant decrease $(\sim 30 \%)$ in the total lipid content in aged $\left(0.120 \pm 0.13 \mu \mathrm{g} / \mathrm{cm}^{2}\right)$ vs young $\left(0.177 \pm 0.02 \mu \mathrm{g} / \mathrm{cm}^{2} ; P<0.05\right)$ murine stratum corneum (Fig. 2 ). In contrast, the lipid distribution of senescent vs young murine stratum corneum showed a similar profile and distribution of the major neutral lipids (free sterols, sterol esters, free fatty acids, and triglycerides) and sphingolipid species, including acylceramides (ceramide 1) (Table I). These results indicate that epidermal aging is accompanied by a global decrease in stratum corneum lipid content, resulting in a comparable reduction of each of the major lipid species. However, no selective reduction of any specific lipid species occurs under basal conditions.

Fatty acid composition of senescent epidermal lipids. We next examined the fatty acid distribution of the combined neutral and sphingolipid species to determine whether a decrease in either essential fatty acids or in very long chain species exists in aged epidermis. As seen in Fig. 3 the spectrum of fatty acids in senescent murine epidermal lipids does differ from young controls, i.e., it displays a disproportionate increase in medium and long chain (C14-C18) species. Moreover, there is no corresponding decrease in any of the very long chain species (C20C24), or in linoleic acid (quantitative data not shown). Thus, analysis of the constituent fatty acids of the epidermal lipids does not reveal any significant abnormality in aging epidermis under basal conditions.

\section{Dynamic studies of aged epidermal barrier function}

Barrier integrity. We next assessed the integrity of the stratum corneum in aged vs young subjects. Whereas only $18 \pm 2$ tape strippings were required to perturb the barrier of the volar forearms of aged human subjects, a significantly greater number of tape strippings $(31 \pm 5 ; P<0.05)$ were required to perturb the barrier over comparable sites in the younger human subjects. Although difficult to compare accurately because of differences in each investigator's technique, and because different amounts of acetone may actually contact the test area, only 3-10 min of acetone soaking was required for barrier perturbation in the aged vs 30-60 min to perturb the barrier in the young. Likewise, the barrier in aged mice was more readily disrupted by tape stripping than in younger mice ( $\sim 3$ vs $\sim 5$ strippings, respectively, for aged vs young animals). These studies demonstrate that, while barrier function appears to be normal under basal conditions, the integrity of the stratum corneum is diminished in chronologically aged epidermis.

Barrier recovery. To determine whether the measurements of TEWL under basal conditions masked an underlying abnormality in barrier function, we next compared the rate of barrier recovery in aged vs young humans and hairless mice. After comparable insults (TEWL $20-30 \mathrm{~g} / \mathrm{m}^{2}$ per $\mathrm{h}$ ) the epidermis of aged and young each display a barely detectable shiny appearance, with no obvious damage to the epidermis. This change is no longer visible in either young or old at subsequent time points. Despite subnormal TEWL rates under basal conditions, barrier recovery after comparable insults was much slower in aged than in young humans, regardless of the mode of barrier insult, i.e., acetone treatment or tape stripping (Fig. $4, A$ and $B$, respectively). For example, whereas the aged human barrier 

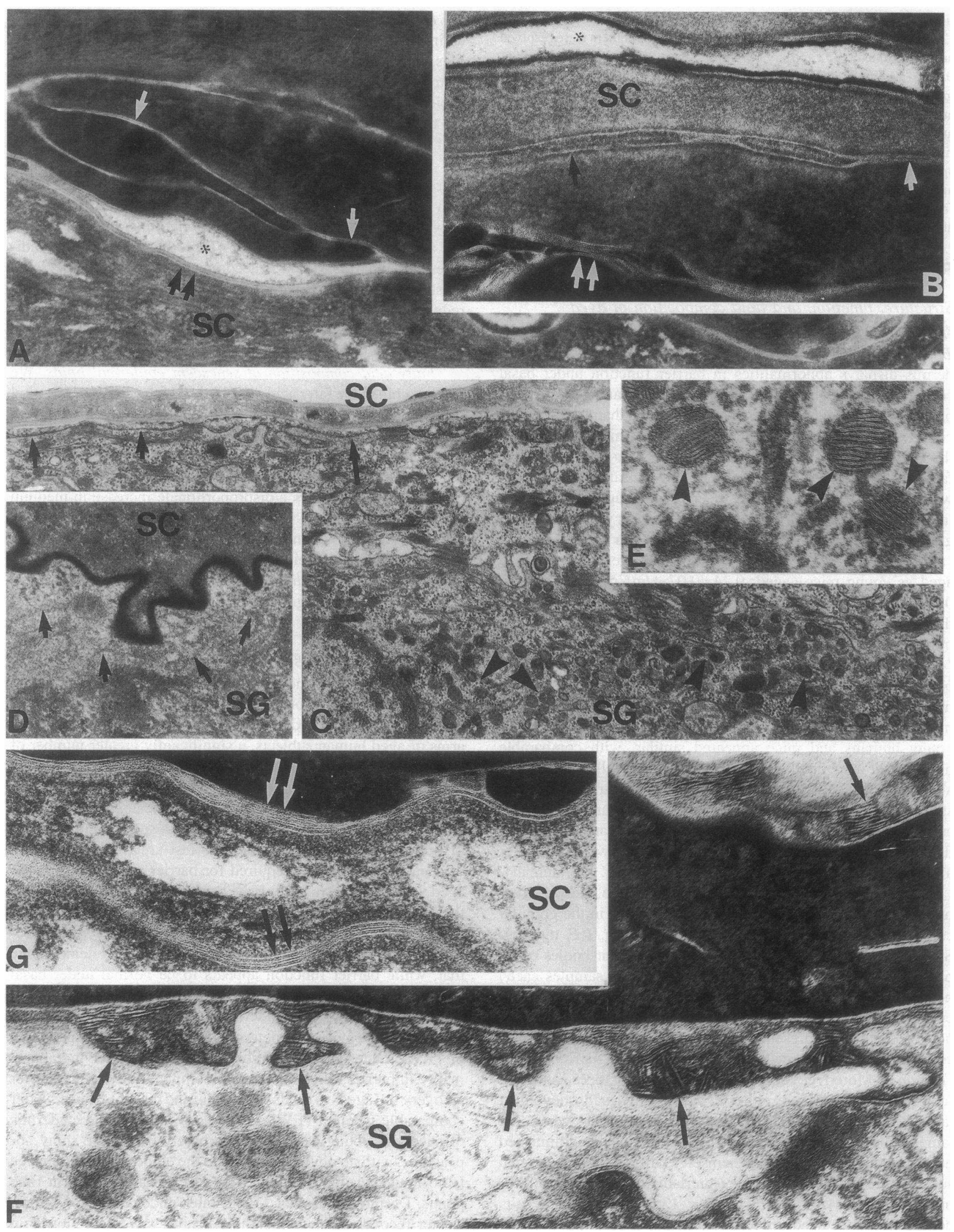


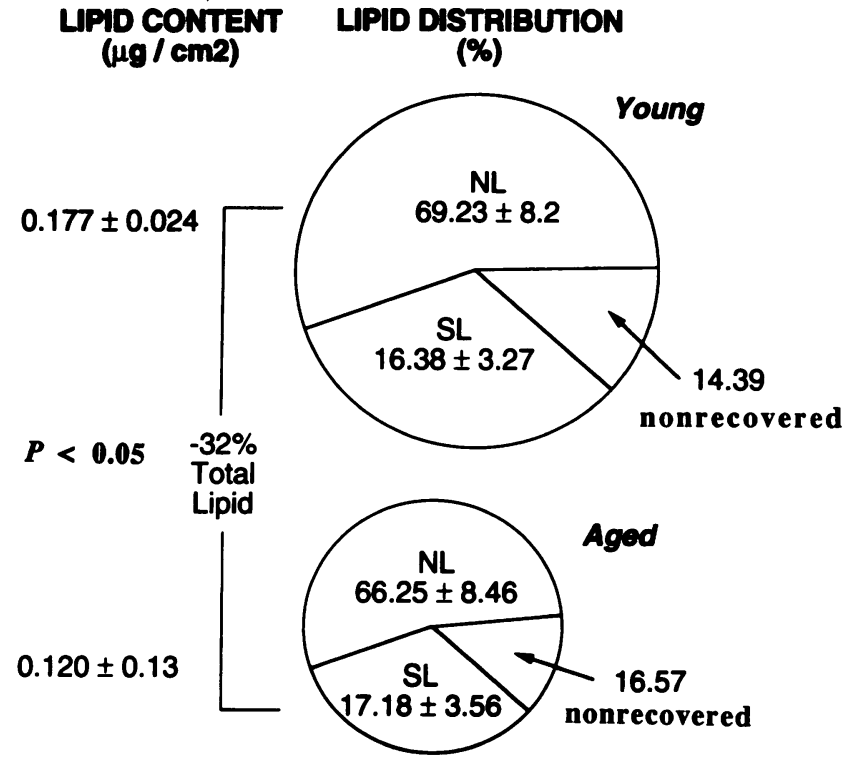

Figure 2. Lipid content of aged murine stratum corneum. Total lipid content was decreased by $32 \%$ in aged murine stratum corneum vs young stratum corneum. However, the lipid species distribution remained unchanged. Data shown are mean \pm SEM. $n=6$.

only recovered by $15 \%$ at $24 \mathrm{~h}$ after acetone treatment, barrier function in the younger subjects typically recovered by $50 \%$ ( $P$ $<0.01$ ). Moreover, whereas the younger barrier recovered by $\sim 70-80 \%$ by $48 \mathrm{~h}, 5-6 \mathrm{~d}$ were required for aged skin to attain this level of barrier recovery regardless of the type of insult. In the aged the barrier had recovered by $>90 \%$ by $7 \mathrm{~d}$, whereas in the young, $>90 \%$ recovery was seen by $4 \mathrm{~d}$. Barrier recovery in aged vs young hairless mice (Fig. 5) displayed a similar difference, although both age groups recovered over shorter time periods than did human subjects. For example, while the aged barrier only recovered by $15 \%$ by $6 \mathrm{~h}$, it recovered by $50 \%$ in younger animals (Fig. $5, P<0.001$ ). These results demonstrate a profound abnormality of barrier function in aged epidermis that only becomes apparent when the organ is stressed, i.e., following barrier disruption.

Stratum corneum neutral lipid distribution (Fig. 6). To determine the structural basis for the functional abnormalities in aged epidermis, we next assessed the distribution of stratum corneum lipids before and after barrier disruption. Immediately before barrier disruption, a similar pattern of neutral lipid staining was seen in the stratum corneum of both aged and young hairless mice (Fig. 6, $A$ and $B$ ). Moreover, immediately after acetone wiping, histochemical staining for neutral lipids using sudan red 7B was absent in both young and old murine stratum
Table I. Lipid Distribution in Aged vs Young Murine Stratum Corneum Lipid Content

\begin{tabular}{lcc}
\hline & Young & Aged \\
\hline & \%total lipid \pm SEM & \%total lipid \pm SEM \\
Total sphingolipid & $16.38 \pm 3.27$ & $17.78 \pm 3.56$ \\
Acylceramide & $7.52 \pm 1.38$ & $8.18 \pm 1.56$ \\
Ceramide III & $2.30 \pm 0.42$ & $2.30 \pm 0.34$ \\
Ceramide IV & $2.43 \pm 0.66$ & $2.48 \pm 0.41$ \\
Acylglucosylceramide & $0.82 \pm 0.35$ & $0.80 \pm 0.42$ \\
Total neutral lipid & $69.23 \pm 8.2$ & $66.25 \pm 8.46$ \\
Total identified lipid & $85.61 \pm 5.68$ & $84.0 \pm 2.53$ \\
& & \\
\hline
\end{tabular}

corneum. Whereas at $3 \mathrm{~h}$ no visible return of lipid could be seen yet in either young or old stratum corneum, by 6 and 16 h significant amounts of stainable neutral lipid material had reappeared in young murine stratum corneum (Fig. 6, $C$ and $E$, respectively). In contrast, staining did not return to aged murine stratum corneum until after $16 \mathrm{~h}$ (Fig. 6, D-G). By 24 h equivalent amounts of lipid appeared to have reaccumulated in the stratum corneum of both young and aged stratum corneum (not shown), and both were indistinguishable from untreated controls. A similar delay in reappearance of neutral lipids was seen in frozen sections of senescent murine stratum corneum stained with the hydrophobic fluorophore, nile red, at $6 \mathrm{~h}$ (data not shown). These results show that the abnormality in barrier recovery in aged murine epidermis is accompanied by a corresponding delay in the reappearance of stainable neutral lipids in the stratum corneum.

Lamellar body secretory response to barrier disruption. Electron microscopy with osmium tetroxide after fixation was used to determine the lamellar body response to barrier disruption. $3 \mathrm{~h}$ after barrier disruption large numbers of newly formed lamellar bodies, filled with abundant lipid, were visible in the stratum granulosum of young murine epidermis (data not shown; see reference 39). In the aged there also was abundant lamellar body formation, but these structures were not replete with lipid and many contained only a few bilayer structures (Fig. 7). Furthermore, after $3 \mathrm{~h}$, while the stratum granulosumstratum corneum interface in the young contained extensive lipid material (see reference 39), the stratum granulosumstratum corneum interface in the aged is still devoid of lamellar material (Fig. 7).

\section{Discussion}

Because permeability barrier function in aging epidermis does not appear to be impaired under basal conditions, it has been

Figure 1. $(A-E)$ Aged human and murine epidermis. $A$ and $B$ demonstrate abnormalities in intercellular lamellae in the stratum corneum ( $S C$ ) of two different aged human subjects. Note decreased number of lamellae along extended membrane regions (single arrows), although in some areas normal numbers of lamellae are present (double arrows). Extensive intercellular clefts (asterisks) are also more common in aged than in young $S C$. $C-E$ demonstrate normal numbers of lamellar bodies ( $C$, arrowheads) in aged stratum granulosum ( $S G$ ), and lamellar bodies have normal internal structure ( $E$, arrowheads) ( $C$ and $E$, human; $D$, mouse). However, the quantity of secreted lamellar body contents at the $S G-S C$ interface appears to be reduced in both aged human $(C$, single arrows) and murine epidermis $(D$, single arrows depict apical plasma membrane of $S G$ cell with no evidence of secreted intercellular lamellae). $F$ and $G$ depict young human epidermis for comparison. Note abundant intercellular lamellae in $S C(G$, double arrows) and secreted lamellar body contents at $S G-S C$ interface $(F$, single arrows $) . A, \times 55,000 ; B, \times 60,000, C, \times 21,000 ; D$, $\times 58,000 ; E, \times 94,000 ; F, \times 72,000$; and $G, \times 106,000$. $C-E$, osmium tetroxide; $A, B, F$, and $G$, ruthenium tetroxide. 

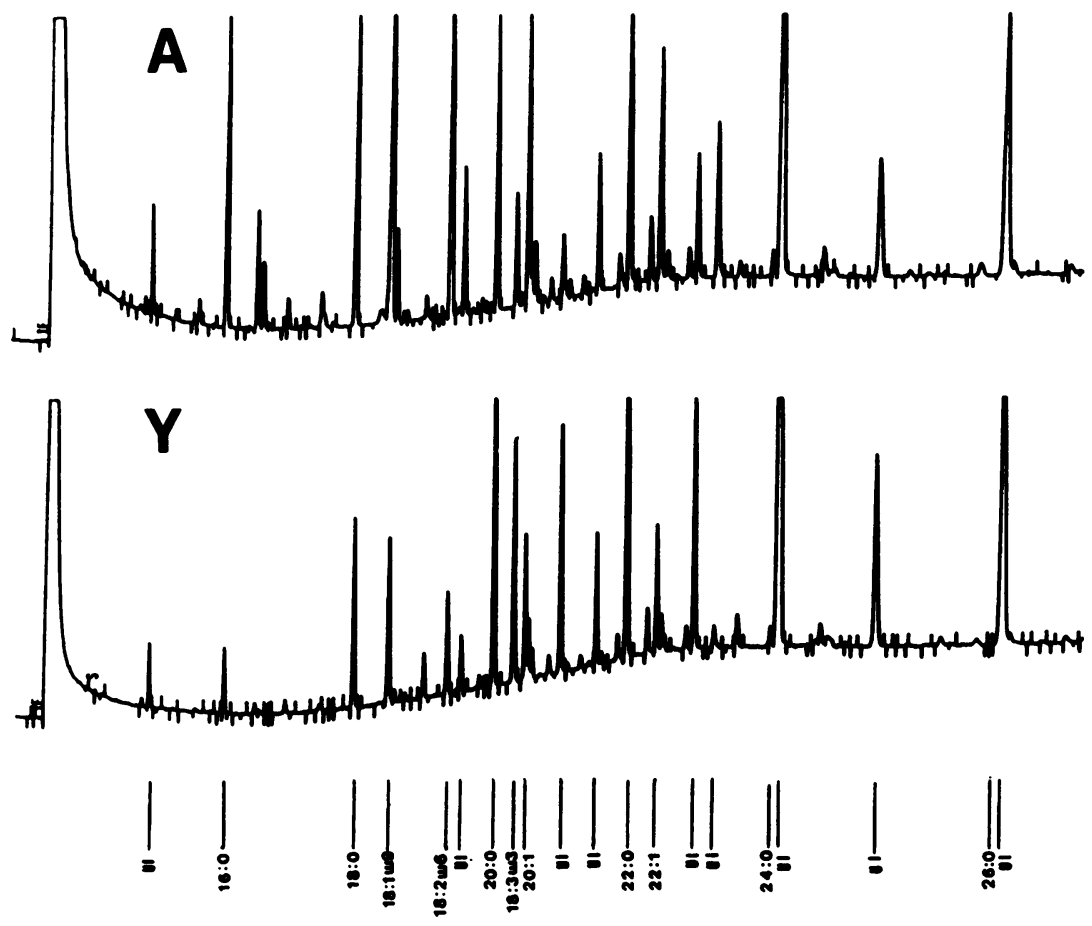

Figure 3. Fatty acid content of senescent epidermal lipids. There is a disproportionate increase in medium and long chain $(\mathrm{Cl}-\mathrm{Cl})$ species in aged $(A)$ vs young $(Y)$ murine epidermis. However, no changes are apparent in very long chain species $(C 20-C 24)$, nor in linoleic acid $(C 18)$. generally assumed that barrier function is not altered significantly in aging (40). The cause of the reduced rates of TEWL of aged epidermis under basal conditions are unknown. Possible causes of a decreased TEWL could include decreased sweating, decreased microcirculation resulting in decreased integumental moisture content, decreased skin temperature, or increased corneocyte cell surface area. Although neither decreased sweating (41), decreased microvasculature (42), nor decreased skin surface temperature (14) alone can account for the decreased TEWL in aged epidermis. A combination of these factors could be operative. In contrast, it has been long known that aged epidermis is abnormally permeable to a variety of drugs in in vitro systems $(14-16)$. Thus, it seemed likely to us that the seemingly normal and even subnormal levels of TEWL displayed by intrinsically aged epidermis might mask an underlying lack of functional reserve. The functional, structural, and lipid biochemical studies shed light on this apparent paradox. When the epidermal permeability barrier is subjected to stress, i.e., barrier abrogation, marked differences in the function of intrinsically aged vs young stratum corneum appear.

In seeking the basis for the functional abnormality, we found that both aged human and mouse epidermis displayed a normal number of lamellar bodies in the granular cell cytosol with normal internal structure. However, a paucity of secreted lamellar body contents was present at the stratum granulosum-stratum corneum interface. Moreover, the stratum corneum interstices contained focal domains that were either depleted or virtually devoid of lamellar structures. Yet, although diminished in numbers, the lamellar arrays themselves possessed a normal substructure. These findings match the lipid biochemical observations which showed that stratum corneum lipids in aging are globally reduced in quantity, without exhibiting any specific abnormality in species distribution or fatty acid composition. It is difficult to compare our results with prior reports because most of these studies only sampled the superficial stratum cor- neum (surface lipids) with either solvents or tape stripping (e.g., 18, 19). One of these workers reported a decrease in sterol esters and triglycerides with aging (18). In another recent study, only ceramide content was assessed (19), but this study, using a single cyanoacrylate stripping of stratum corneum, demonstrated a decrease in the ceramide content in normal humanaged skin. These workers then correlated the decrease in ceramide content with a decrease in sphingomyelinase activity (43). We, too, found that ceramides were decreased. However, the decrease was not specific for sphingolipids, but instead extended to each of the major lipid species analyzed.

Whereas these studies have shown that baseline functional parameters are only minimally altered in aged skin, dynamic assessments revealed profound abnormalities in the functional capacity of the aged epidermal permeability barrier. For example, barrier integrity, as indicated by resistance to sequential tape stripping or acetone wiping, is markedly reduced in aging epidermis. Aged stratum corneum has been noted previously to exhibit altered cohesiveness (44), which could result in increased amounts of material obtained with each tape stripping. The decreased integrity of the barrier in intrinsically aged stratum corneum may relate to this altered cohesiveness, which in turn, could be due to the decreased intercellular lipid content that we have described here. Whether the decreased lipid content and focal abnormalities in the intercellular spaces of aged stratum corneum either account for or contribute to the abnormality in integrity remains speculative.

In addition to altered integrity, barrier recovery is delayed in aged human and murine skin after barrier abrogation by either acetone or tape stripping. Delipidization or removal of the epidermal permeability barrier is followed by a rapid phase of barrier recovery over $6 \mathrm{~h}$ in young hairless mice, followed by a slower recovery phase requiring $30-36 \mathrm{~h}$ to reach completion (45). Whereas the acute recovery phase stimulates epidermal cholesterol and fatty acid synthesis (46), the late recovery 

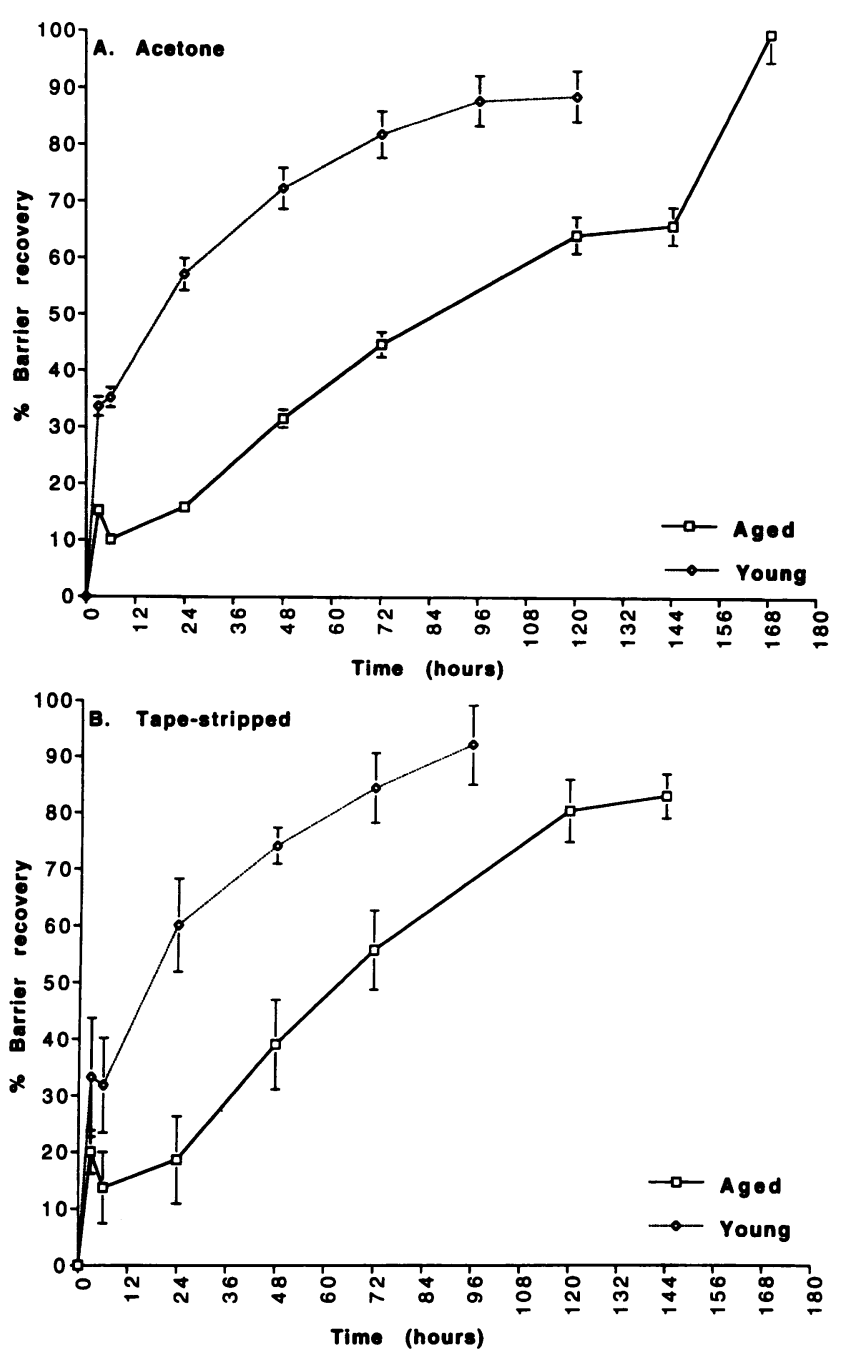

Figure 4. Human barrier recovery. (A) Human barrier recovery after acetone wiping: recovery is delayed in the aged $(P<0.01$ at all time points). Note that at $24 \mathrm{~h}$ the aged barrier is recovered only $15 \%$ while the young barrier is almost $50 \%$ recovered. $(B)$ Human barrier recovery after tape stripping reveals a similar divergent pattern of barrier recovery in aged vs young human epidermis. Data shown are mean \pm SEM at each time point. $n=5$.

phase is accompanied by increases in sphingolipid (47) and DNA (24) synthesis. This burst in synthetic activity is accompanied by a return of lipid to the stratum corneum interstices, as shown by histochemical techniques (45), and an early burst in lamellar body synthesis and secretion, as seen by electron microscopy (39). Thus, the aged epidermal permeability barrier is both easier to perturb and slower to repair. This is a consistent finding in both humans and mice, using two different methods of acute barrier perturbation. The use of both acetone and tape stripping parallel studies allowed us to control for the possible unwanted effects of each model. Hence, neither cytotoxicity from acetone, nor the added variable of cellular replacement with tape stripping are likely to account for our results. The basis for the delay in barrier recovery in aging epidermis appears to be a delay in the return of stainable neutral lipids to the stratum corneum interstices, as demonstrated by histochemistry and electron microscopy. The delay in lipid reaccumulation, in

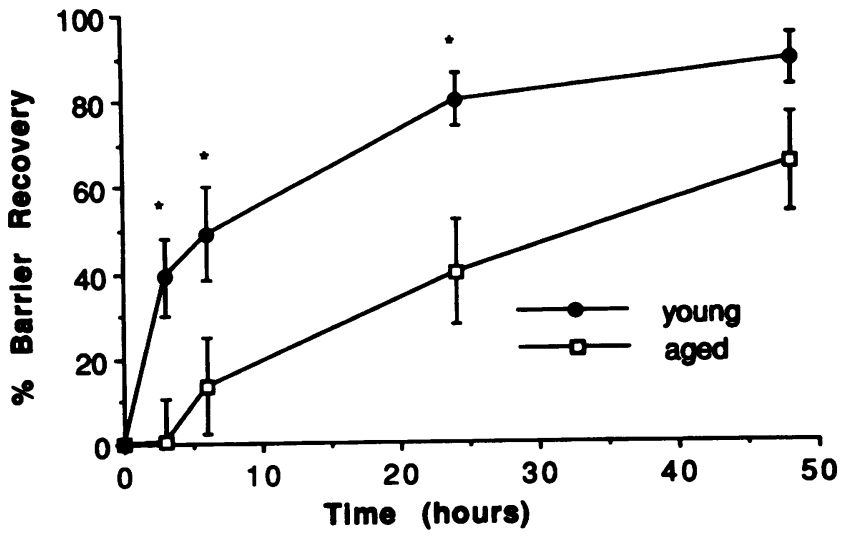

Figure 5. Murine barrier recovery. Murine barrier recovery after tape stripping is delayed in aged vs young epidermis $(P<0.01)$. Recovery takes place with a similar pattern to human epidermis (compare with Fig. 4), but over a shorter time period than in humans. Data shown are mean \pm SEM at each time point. $n=10$.

turn, may be attributed to diminished lamellar body secretion, and therefore a decrease in lipid delivery to the stratum corneum interstices. The retardation in lipid delivery could lead to a delay in the normal rate of lamellar bilayer reformation in the intercellular spaces. Thus, when the epidermal permeability barrier is subjected to stress, i.e., barrier abrogation, there are marked differences in the function of intrinsically aged vs young stratum corneum. Our results show further that measurements of basal TEWL do not reflect the actual functional status of the tissue, and can even be misleading. In this respect, skin, as is the case with other organs (21-23), may display profound abnormalities only when the organ is stressed or undergoes repair processes. Most importantly, measurements of basal function provide no insights about the capacity of the epidermis to maintain barrier homeostasis when subjected to injury, chemical exposure, or environmental stress.

The metabolic basis for the biochemical and structural abnormalities in aging stratum corneum is not known. Our previous studies have shown that an acute burst in both lipid and DNA synthesis follows barrier abrogation (reviewed in 46). Moreover, epidermis possesses the capacity to synthesize a full spectrum of lipids, including ceramides (48) cholesterol (49), and free fatty acids (46). The synthesis of all three key lipids is not only modulated by barrier requirements (46), but also required for barrier homeostasis $(27,50)$. The burst in lipid biosynthesis after experimental perturbation leads to lipid replenishment in the stratum corneum, which is followed by a return of barrier function (45). The increases in cholesterol, ceramide, and fatty acid synthesis that accompany barrier repair result from a corresponding increase in the total activity of the rate-limiting enzymes for these lipids, HMG CoA reductase (25), serine palmitoyl transferase (26), and acetyl CoA carboxylase (unpublished data). Recent studies also have shown that barrier disruption induces a rapid increase in the mRNA for HMG CoA reductase (51), as well as changes in the phosphorylation state of this enzyme (25). Future studies should be able to determine whether the decreased ability of aged epidermis to repair the barrier can be attributed to specific or global lipid metabolic abnormalities, and to determine the molecular bases for these alterations. 

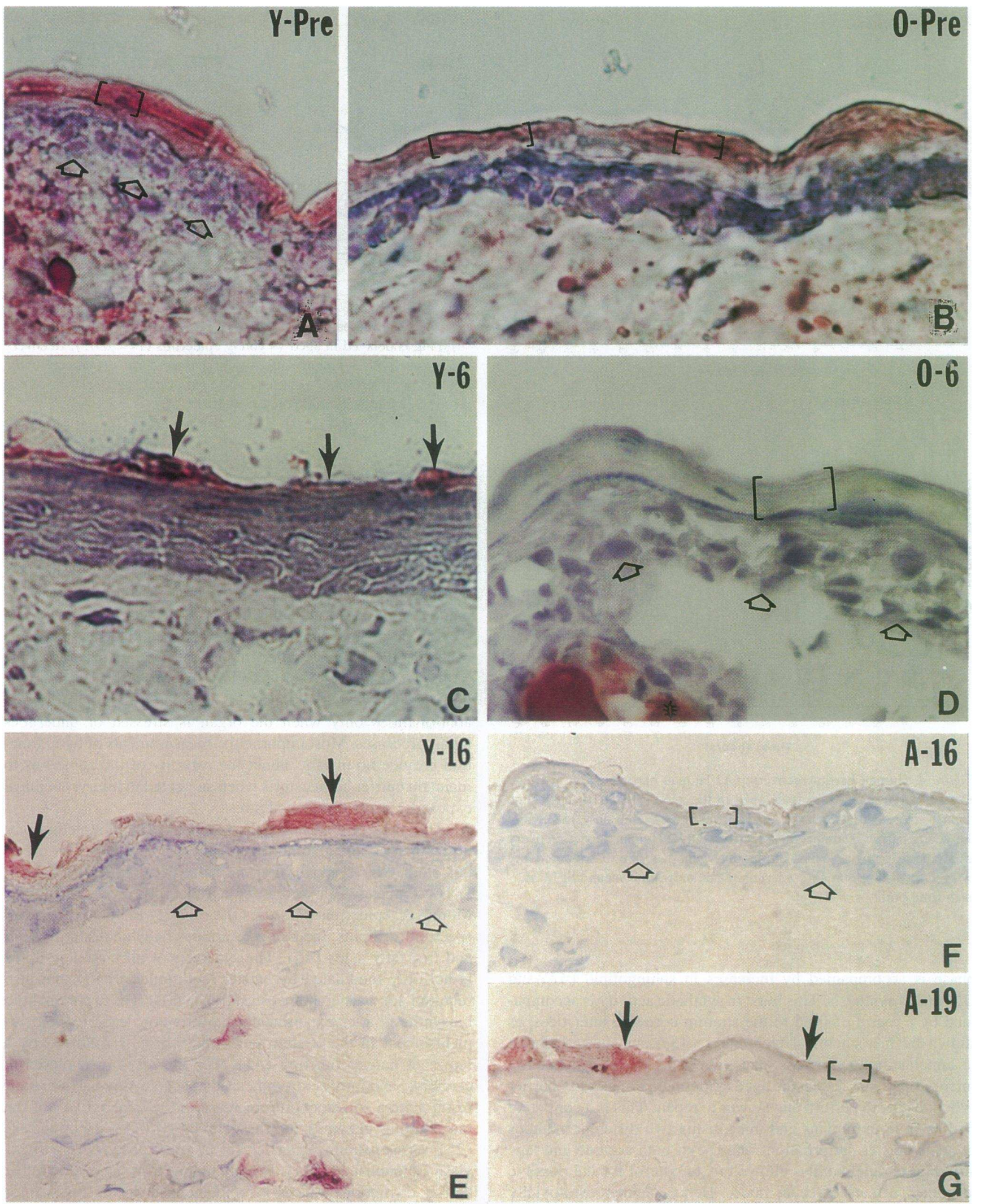

Figure 6. Histochemical localization of neutral lipids in young $(Y)$ vs old $(O)$ murine epidermis. $A$ and $B$ are before and $C-G$ after acetone treatment. Immediately before acetone treatment the stratum corneum ( $A$ and $B$, brackets) of both young and old reveals abundant neutral lipid. By $6 \mathrm{~h}$ after acetone treatment, substantial neutral lipid has returned to the stratum corneum of young animals $(C$, arrows), while old stratum corneum displays no return of neutral lipids $(D$, brackets $)$. By $16 \mathrm{~h}$, more neutral lipid staining has appeared in young stratum corneum ( $E$, arrows), but aged stratum corneum at $16 \mathrm{~h}$ still displays virtually no return of neutral lipid $(F$, brackets $)$. Only at $19 \mathrm{~h}$, does stainable lipid begin to appear in stratum corneum of aged mice $(G$, brackets and arrows). Note intense staining of the sebaceous glands, which serve as a positive control. Open arrows indicate basement membrane in A, D-F. $A-G, \times 400$. 


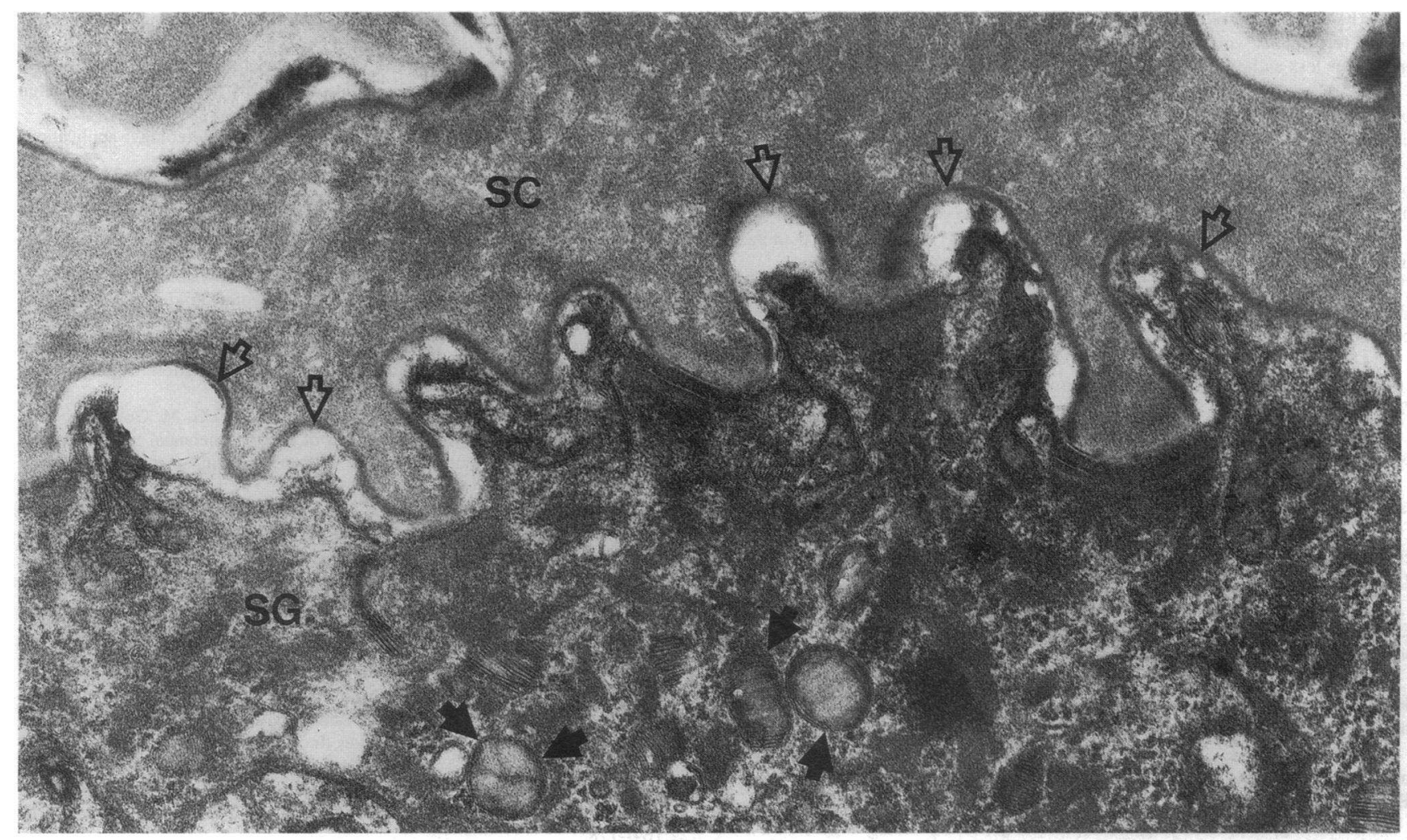

Figure 7. Lamellar body response to murine barrier disruption. $3 \mathrm{~h}$ after barrier disruption in the aged the stratum granulosum-stratum corneum junction is devoid of lamellar structures (open arrows). Although newly formed lamellar bodies are evident, their contents are diminished or virtually absent (closed arrows). $\times 75,000$.

Finally, we have described here a new murine model of cutaneous senescence. The Charles River hairless mouse strain displays a normal, murine life expectancy without the premature development of neoplasms, a common occurrence with other commercially available, inbred, hairless mouse strains. By all parameters examined to date, the epidermal abnormalities in this model parallel those that occur in the intrinsically aged epidermis of humans of advanced age ( $>80 \mathrm{yr}$ ). In both species, we did not find consistent abnormalities in individuals of less advanced age (humans $<80 \mathrm{yr}$; mice $<14 \mathrm{mo}$; Ghadially, R. and P. M. Elias, unpublished observations.). Thus, the changes described in this report are detected only with relatively advanced senescence. Whether the program of chronologic senescence is accelerated when chronic photodamage is superimposed upon intrinsic aging remains to be determined.

In conclusion, in aged skin, functional assessments under basal conditions do not provide an accurate assessment of epidermal function. In contrast, stress to the system reveals underlying alterations in epidermal integrity and barrier recovery after experimental perturbation. A global decrease in lipid content leads to alterations in lamellar bilayer morphology which appear to underlie the impaired functional abnormalities. In addition, we have described a new murine model of skin aging which may be useful for future animal studies to elucidate the biochemical mechanisms responsible for epidermal aging. Finally, these findings have potential clinical significance. First, the decrease in lipid content may contribute to the commonly xerotic skin of the aged. Second, the decreased barrier integrity implies an increased susceptibility to the insults of the environment (e.g., decreased humidity, solvents, and detergents ), as well as a diminished capacity to recover from environmental insult.

\section{Acknowledgments}

We gratefully acknowledge the excellent technical assistance of Debra A. Crumrine and Deborah Lawson, M.D.

This work was supported by the Dermatology Foundation, the Veterans Affairs Medical Research Service, the Lester I. Conrad Research Foundation, and National Institutes of Health grants AR19098, AR 39448, and AR 39639.

\section{References}

1. Christopher, E., and A. M. Kligman. 1965. Percutaneous absorption in aged skin. In Advances in Biology of the Skin. W. Montagna editor. Pergamon Press, Inc. New York. 163-175.

2. Grove, G. L., S. Duncan, and A. M. Kligman. 1982. Effect of aging on the blistering of human skin with ammonium hydroxide. Br. J. Dermatol. 107:393400.

3. Lavker, R. 1979. Structural alterations in exposed and unexposed skin. $J$. Invest. Dermatol. 73:259-266.

4. Lavker, R., P. Zheng, and G. Doug. 1987. Aged skin: a study of light, transmission electron, and scanning electron microscopy. J. Invest. Dermatol. $88: 44 \mathrm{~s}-51 \mathrm{~s}$

5. Black, M. 1969. A modified radiographic method for measuring skin thickness. Br. J. Dermatol. 81:661-666.

6. Shuster, S., and M. M. Black. 1975. The influence of age and sex on skin thickness, skin collagen and density. Br. J. Dermatol. 93:639-643.

7. Marks, R. 1981. Measurement of biological aging in human epidermis. Br. J. Dermatol. 104:627-633.

8. Thune, P. 1989. Evaluation of the hydration and the water-holding capacity in atopic skin and so-called dry skin. Acta Dermato-Venereol. (Suppl.) 144:133135. 
9. Elsner, P., D. Wilhelm, and H. I. Maibach. 1990. Frictional properties of human forearm and vulvar skin: influence of age and correlation with transepidermal water loss and capacitance. Dermatologica (Basel). 181:88-91.

10. Wilhelm, K.-P., A. B. Cua, and H. I. Maibach. 1991. Skin aging. Effect on transepidermal water loss, stratum corneum hydration, skin surface $\mathrm{pH}$, and casual sebum content. Arch. Dermatol. 127:1806-1809.

11. Cua, A. B., K.-P. Wilhelm, and M. I. Maibach. 1990. Frictional properties of human skin: relation to age, sex and anatomical region, stratum corneum hydration and transepidermal water loss. Br. J. Dermatol. 123:473-479.

12. Potts, R. O., E. M. Buras, and D. A. Christman. 1984. Changes with age in the moisture content of human skin. J. Invest. Dermatol. 82:97-100.

13. Menon, G. K., K. R. Feingold, A. H. Moser, B. E. Brown, and P. M. Elias. 1985. De novo sterologenesis in the skin. II. Regulation by cutaneous barrier requirements. J. Lipid Res. 26:418-427.

14. Leveque, J. L., P. Corcuff, J. de Rigal, and P. Agache. 1984. In vivo studies of the evolution of physical properties of the human skin with age. Int. J. Dermatol. 23:322-328.

15. Tagami, H. 1972. Functional characteristics of aged skin. Acta Dermatol. 66:19-21.

16. Malkinson, F. D. 1958. Studies on the percutaneous absorption of $14 \mathrm{C}$ labelled steroid by use of the gas flow cell. J. Invest. Dermatol. 31:19-28.

17. Grubauer, G., K. R. Feingold, R. M. Harris, and P. M. Elias. 1989. Lipid content and lipid type as determinants of the epidermal permeability barrier. $J$. Lipid Res. 30:89-96.

18. Léger, D. S., A. M. François, J. L. Lévêque, T. J. Stoudemayer, G. L. Grove, and A. M. Kligman. 1988. Age-associated changes in stratum corneum lipids and their relation to dryness. Dermatologica (Basel). 177:159-164.

19. Imokawa, G., A. Akihito, J. Kumi, Y. Higaki, M. Kawashima, and A. Hidano. 1991. Decreased level of ceramides in stratum corneum of atopic dermatitis: an etiologic factor in atopic dry skin? J. Invest. Dermatol. 96:523-526.

20. Stewart, M. E., and D. T. Downing. 1991. Chemistry and function of mammalian sebaceous lipids. In Advances in Lipid Research. Skin Lipids. P. M. Elias, editor. Academic Press, New York. 282-283.

21. Katzung, B. G. 1992. Special aspects of geriatric pharmacology. In Basic and Clinical Pharmacology. B. G. Katzung, editor. Appleton \& Lange, East Norwalk, CT. 862-863.

22. Davies, I. 1992. Theoriès and general principles of aging. In Textbook of Geriatric Medicine and Gerontology. 4th ed. J. C. Brocklehurst, R. C. Tallis, and H. M. Fillit, editors. Churchill-Livingstone, Inc., New York. 26-60.

23. Miller, R. A. 1994. The biology of aging and longevity. In Principles of Geriatric Medicine and Gerontology. 3rd ed. W. R. Hazzard, E. L. Bievman, J. P. Blass, W. H. Ettinger, and J. B. Halter, editors. McGraw-Hill, Inc., New York. 3-18.

24. Proksch, E., K. R. Feingold, M. Mao-Qiang, and P. M. Elias. 1991. Barrier function regulates epidermal DNA synthesis. J. Clin. Invest. 87:1668-1673.

25. Proksch, E., P. M. Elias, and K. R. Feingold. 1990. Regulation of 3hydroxy-3-methyl-glutarylcoenzyme A reductase activity in murine epidermis: modulation of enzyme content and activation state by barrier requirements. $J$. Clin. Invest. 85:874-882.

26. Holleran, W. M., K. R. Feingold, M. Mao-Qiang, W. N. Gao, J. M Lee, and P. M. Elias. 1991. Regulation of epidermal sphingolipid synthesis by permeability barrier function. J. Lipid Res. 32:1151-1158.

27. Ottey, K., L. C. Wood, and P. M. Elias. 1995. Effect of cutaneous permeability barrier distruption on fatty acid synthetic enzymes in the epidermis of hairless mice. J. Lipid Res. In press.

28. Blichmann, C. W., and J. Serup. 1987. Reproducibility and variability of transepidermal water loss measurement. Acta Dermato-Venereol. 67:206-210.

29. Ghadially, R. G., L. Halkier-Sorensen, and P. M. Elias. 1992. Effects of petrolatum on stratum corneum structure and function. J. Am. Acad. Dermatol. 26:387-396.

30. Hou, S. Y. E., A. K. Mitra, S. H. White, G. K. Menon, R. Ghadially, and P. M. Elias. 1991. Membrane structures in normal and essential fatty acid deficient stratum corneum: characterization by ruthenium tetroxide staining and $\mathrm{x}$-ray diffraction. J. Invest. Dermatol. 96:215-223.

31. McNutt, N. S., and W. L. Crain. 1981. Quantitative electron microscope comparison of lymphatic nuclear contours in mycosis fungoides and in benign infiltrates of the skin. Cancer (Phila.). 47:163-166.

32. Elias, H., A. Henning, and D. Schwartz. 1971. Stereology: applications in biomedical research. Physiol. Rev. 51:158-200.

33. Menon, G. K., S. K. Aggarwal, and A. M. Lucas. 1981. Evidence for the holocrine nature of lipid secretion in avian epidermal cells. A histochemical and fine structural study of the rictus and the uropyogial gland. J. Morphol. 167:185199.

34. Fowler, S. D., and P. H. Greenspan. 1985. Application of nile red, a fluorescent hydrophilic probe for the detection of neutral lipid deposits in tissue sections. J. Histochem. Cytochem. 33:833-836.

35. Lampe, M. A., M. L. Williams, and P. M. Elias. 1983. Human epidermal lipids: characterization and modulations during differentiation. J. Lipid Res. 24:131-140.

36. Elias, P. M., B. E. Brown, P. Fritsch, J. Goerke, G. M. Gray, and R. J. White. 1979. Localization and composition of lipids in neonatal mouse stratum granulosum and stratum granulosum and stratum corneum. J. Invest. Dermatol. 73:339-348.

37. Bligh, E. G., and W. J. Dyer. 1959. A rapid method of total lipid extraction and purification. Can. J. Biochem. Physiol. 37:911-917.

38. Ponec, M., A. Weerheim, J. Kempenaar, A. M. Mommaas, and D. H. Nugteren. 1988. Lipid composition of cultured human keratinocytes in relation to their differentiation. J. Lipid Res. 29:949-961.

39. Menon, G. K., K. R. Feingold, and P. M. Elias. 1992. Lamellar body secretory response to barrier disruption. J. Invest. Dermatol. 98:279-289.

40. Wilhelm, K. P., and H. I. Maibach. 1993. Influence of aging on the barrier function of human skin evaluated by in vivo transepidermal water loss measurements. In Aging Skin: Properties and Functional Changes. J. L. Leveque and P. G. Agache, editors. Marcel Dekker, Inc. New York. 239-250.

41. Pinnagoda, J., R. A. Tupker, T. Agner, and J. Serup. 1990. Guidelines for transepidermal water loss (TEWL) measurement. A report from the standardization group of the European Society of Contact Dermatitis. Contact Dermatitis. 22:164-178.

42. Roskos, K., and H. I. Maibach. 1992. Percutaneous absorption and age. Implications for therapy. Drugs \& Aging. 2:432-449.

43. Yamamura, T., and T. Tezuka. 1989. Change in sphingomyelinase activity in human epidermis during aging. J. Dermatol. Sci. 10:617-641.

44. Long, C. C., and R. Marks. 1992. Stratum corneum changes in patients with senile pruritus. J. Am. Acad. Dermatol. 27:560-564.

45. Grubauer, G., P. M. Elias, and K. R. Feingold. 1989. Transepidermal water loss; the signal for recovery of barrier structure and function. J. Lipid Res. 30:323-333.

46. Elias, P. M., and K. R. Feingold. 1992. Lipids and the epidermal water barrier: metabolism, regulation and pathophysiology. Semin. Dermatol. 11:176182.

47. Holleran, W. M., M. Mao-Qiang, W. N. Gao, G. K. Menon, P. M. Elias, and K. R. Feingold. 1991. Sphingolipids are required for mammalian epidermal barrier function. Inhibition of sphingolipid synthesis delays barrier recovery after acute perturbation. J. Clin. Invest. 88:1338-1345.

48. Berardesca, E., N. Farinelli, G. Rabbiosi, and H. I. Maibach. 1991. Skin bioengineering in the noninvasive assessment of cutaneous aging. Dermatologica (Basel). 182:1-6.

49. Feingold, K. R., B. E. Brown, S. R. Lear, A. H. Moser, and P. M Elias. 1983. Localization of de novo sterologenesis in mammalian skin. J. Invest. Dermatol. 81:365-369.

50. Feingold, K. R., M. Mao-Qiang, G. K. Menon, S. S. Cho, B. E. Brown, and P. M. Elias. 1990. Cholesterol synthesis is required for cutaneous barrier function in mice. J. Clin. Invest. 86:1738-1745.

51. Jackson, S. M., L. C. Wood, S. Lauer, J. M. Taylor, A. D. Cooper, P. M. Elias, and K. R. Feingold. 1992. Effect of cutaneous permeability barrier disruption on HMG CoA reductase, LDL receptor and apoliprotein E mRNA levels in the epidermis of hairless mice. J. Lipid Res. 33:1307-1314. 